\title{
Sistema de ventilación para labores subterráneas de la empresa PRODUMIN S.A.
}

Ventilation system for underground work of the Company PRODUMIN S.A.

Marco Antonio Mejía Flores. ${ }^{1}$, Karla Margot Morales Chacha. ${ }^{2}$ Diego Ramiro Chacha Borja. $^{3}$

\section{Recibido: 14-03-2020 / Revisado: 19-04-2020 /Aceptado: 12-05-2020/ Publicado: 05-06-2020}

\begin{abstract}
.
DOI: $\underline{\text { https://doi.org/10.33262/concienciadigital.v3i2.2.1253 }}$

The investigation determines a ventilation system for interior mine works in the company PRODUMIN S.A.; The methodology used is to collect data in the field of factors such as: temperature variations, permanent gas emission by blasting and also that established by the machinery. The ranges of dust generation and excess humidity that intervene create unsafe conditions in a work environment, in the same way the deficiency in the operational performance, technical data that was considered with respect to the parameters of the new exploitation front, such as: section of the gallery, machinery to be used, length of the front run and geometry of the mining tasks to be carried out in the new production block. This allows stipulating the most convenient conditions in the area of mining safety of an induced ventilation system compared to the traditionally used ventilation method, and proposing a mixed impeller-aspirating ventilation system with the use of ventilators, extraction turbines and ventilation sleeves.
\end{abstract}

Keywords: Ventilation system, Impeller-aspirant method, Mixed serial method, Underground works.

\section{Resumen.}

La investigación plantea un sistema de ventilación para labores de interior mina en la empresa PRODUMIN S.A.; la metodología utilizada es recoger datos en campo de los factores como: variaciones de temperatura, emisión permanente de gas por voladura y

\footnotetext{
${ }^{1}$ Escuela Superior Politécnica de Chimborazo, Facultad de Recursos Naturales. Riobamba, Ecuador. marco.mejia@espoch.edu.ec

${ }^{2}$ Escuela Superior Politécnica de Chimborazo, Facultad de Recursos Naturales. Riobamba, Ecuador. karla.morales@espoch.edu.ec

${ }^{3}$ Escuela Superior Politécnica de Chimborazo, Facultad de Recursos Naturales. Riobamba, Ecuador. diegoramiroch_88@hotmail.com
} 
también la establecida por la maquinaria. Los rangos de generación de polvo y exceso de humedad que intervienen crean condiciones inseguras en un ambiente de trabajo, del mismo modo la deficiencia en el rendimiento operacional; datos técnicos que fueron considerados con respecto a los parámetros del nuevo frente de explotación, tales como: sección de la galería, maquinaria a utilizarse, longitud de recorrido del frente y geometría de las labores mineras a efectuarse en el nuevo bloque de producción. Esto, permite estipular las condiciones más convenientes en el ámbito de seguridad minera de un sistema de ventilación inducido frente al método de ventilación utilizado tradicionalmente, y proponer un sistema de ventilación mixto impelente-aspirante con el uso de ventiladores, turbinas de extracción y mangas de ventilación.

Palabras claves: Sistema de ventilación, Método impelente-aspirante, Método en serie mixto, Labores subterráneas.

\section{Introducción.}

La Empresa PRODUMIN S.A., es una industria minera dedicada a la explotación y beneficio de minerales metálicos, constituye una de las herramientas más importantes para la promoción del desarrollo social y progreso económico del país. Es así que el contenido de la presente investigación, se enfoca al estudio y conocimiento de la implementación de un sistema de ventilación para labores de interior mina.

Este trabajo se ha realizado aplicando una metodología de campo, para la determinación de factores técnicos y de seguridad industrial minera, como la investigación y propuesta de un sistema de ventilación acorde a las necesidades del nuevo frente de explotación, sustituyendo el sistema tradicional de ventilación que pueden utilizarse para lograr el acondicionamiento del aire que circula a través de las labores subterráneas, teniendo como objetivo principal el proporcionar un ambiente seguro, saludable y en lo posible cómodo para los mineros.

El proyecto que se propone se realiza en dos etapas: La primera radica en el levantamiento de toda la información de campo, y la segunda etapa, se basa en la investigación documental y revisión literaria, de forma que de este modo se pueda identificar los principales problemas enfocados al sistema de ventilación y las medidas que son necesarias poner en práctica, con el fin de controlar y reducir el aire contaminado que circula en labores de interior mina.

\section{Metodología}

La metodología aplicada en el presente trabajo es la metodología de investigación científica exploratoria y una de campo; la primera parte se lo realiza a través de la aplicación del método lógico inductivo, mediante la técnica documental, es decir, por medio de una revisión bibliográfica. Por lo tanto, se ha procedido a una recopilación tanto de artículos como de documentos, relevantes publicados por su importancia en base al tema objeto de estudio. 
La segunda parte consiste, en levantar toda la información de campo que permita identificar los principales problemas enfocados a la ventilación interior mina, y posteriormente realizar los cálculos respectivos con la intención de determinar una ventilación adecuada. Por último, se desarrolla una propuesta de un sistema de ventilación que permite mejorar, tanto las condiciones de los trabajadores, como mejorar los procesos y por ende la producción minera.

\section{Ubicación y posición geográfica}

La empresa PRODUMIN S.A. se encuentra ubicado en el sector "La López" perteneciente al cantón Camilo Ponce Enríquez, provincia del Azuay, dentro de la cooperativa Bella Rica. Las coordenadas UTM de la cooperativa Bella Rica son:
P1: E 641209.00 N 9661650.00
P2: E $643309.00 \mathrm{~N} \quad 9661650.00$
P3: E 643309.00 N 9660250.00
P4: E $646009.00 \mathrm{~N} \quad 9660250.00$
P5: E $646009.00 \mathrm{~N} \quad 9658050.00$
P6: E $641209.00 \mathrm{~N} \quad 9658050.00$

Figura 1:Mapa de Ubicación Geográfica del Operador Minero Produmin S.A.

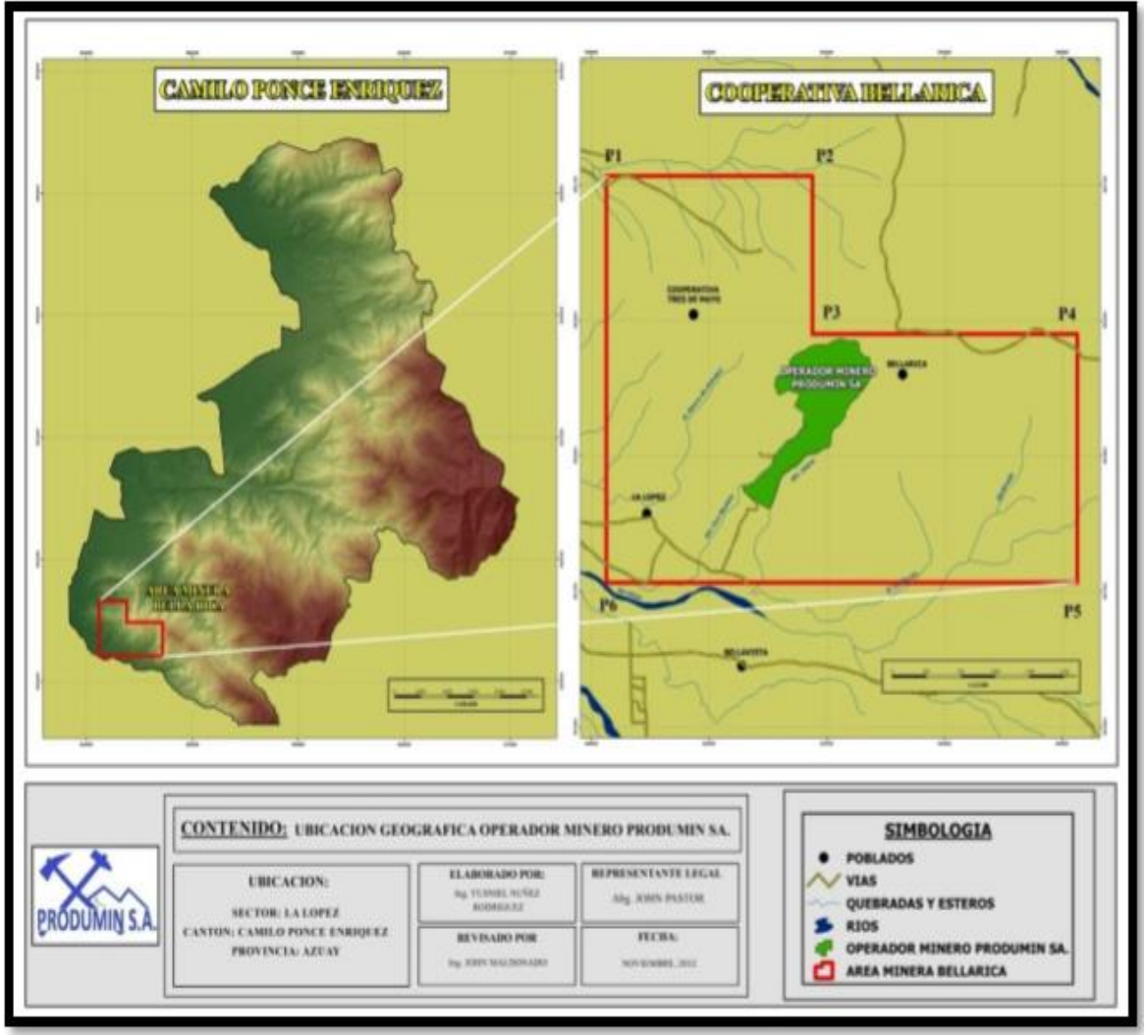

Fuente: Empresa Produmin S.A.

\section{Descripción general de las labores mineras}

Descripción del método de explotación a emplearse en las labores mineras del nuevo frente.- El método de explotación a emplearse es "corte y relleno ascendente convencional con relleno detrítico", es un método donde el estéril y el mineral (veta) son arrancados en 
franjas horizontales por separado, empezando por la parte inferior del tajo y avanzando en forma simultánea verticalmente.

Como labores de preparación a partir de la galería (horizontal) se construyen chimeneas cada de 30m de distancia, tanto para accesos al tajo, "ore pass" y ventilación, en el que su avance es de forma gradual como ascienda el tajeo; dejando un pilar de seguridad de $3 \mathrm{~m}$. Sobre la galería inferior (nivel 0) se construye un subnivel de explotación que comunica las dos chimeneas. Se utiliza máquinas perforadoras Jack-leg con pie de avance para perforaciones verticales y horizontales con taladros de 6 pies de profundidad y un ancho de minado entre 0,80m y un máximo de $1 \mathrm{~m}$ dependiendo del buzamiento y espesor de la veta, desarrollándose hasta dos cortes dependiendo como el comportando del macizo rocoso. La explotación comienza extrayendo una franja horizontal completa de estéril, pero dejando la veta colgada al piso. Posteriormente se somete a voladura la veta colgada y se extrae manualmente con carretillas por las chimeneas trasiego. Se realiza un segundo corte en estéril donde gran parte del material estéril roto se deja como relleno hasta una altura que posibilite perforar nuevamente y se extrae hacia el echadero el excedente formado por el incremento en el volumen de la masa rocosa después del disparo (esponjamiento). Una vez nivelado el piso se procede a realizar la perforación y voladura en veta, para ello se coloca geomembrana en el suelo para que no se diluya el mineral con el estéril del relleno en el piso. El mineral arrancado se extrae por la chimenea trasiego hasta la tolva y posteriormente es transportado a superficie por medio de la locomotora a la tolva de gruesos, para su posterior proceso de trituración. De esta manera se realiza la extracción del mineral y desmonte del tajo. La cual se repetirá hasta llegar al nivel superior del block. (Gutierrez Hijar, 2014)

Gráfico 1: Método de Explotación Corte y Relleno Ascendente-Convencional-Selectivo

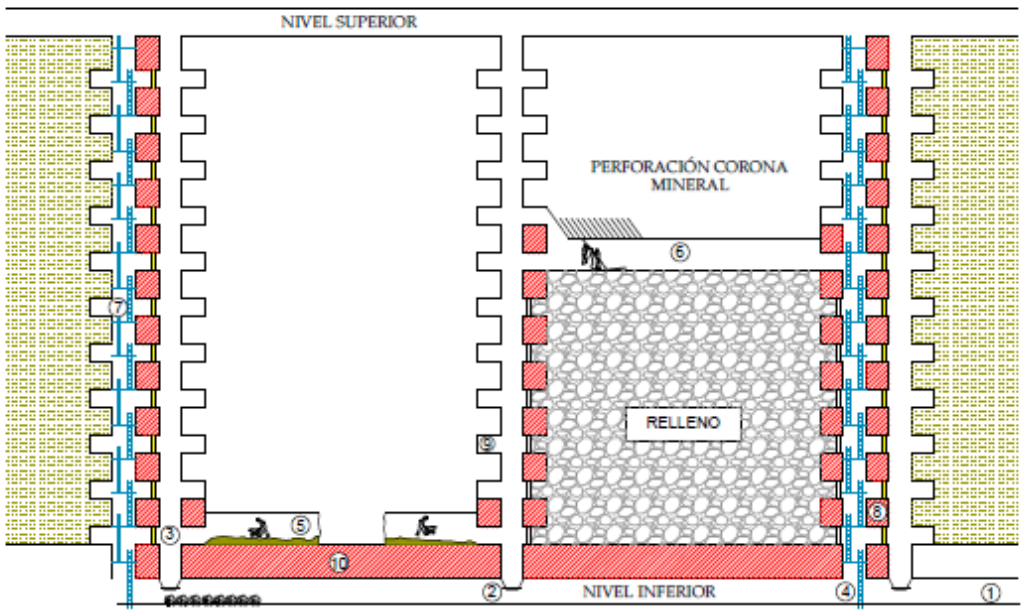

LABORES SUBTERRÁNEAS

1 Galería Principal

2 Tolva

4Chimenes Camino

5 Subnivel

6 Tajo

Rampa de Seguridad

8 Pilar de Segur

10 Puente de Seguridad

Fuente: Empresa Produmin S.A. 


\section{Descripción de la mitigación del polvo en el frente de trabajo con agua}

Para la mitigación del polvo en el frente de trabajo, una vez culminada la etapa de perforación y disparo de la cuña, se mantiene abierta la manguera de aire para acelerar la evacuación de gases y una vez que los mineros lleguen al frente de trabajo se moja el contorno de la galería en el frente de trabajo con la finalidad de asentar el polvo, mejorar la visibilidad y detectar alguna roca suelta. Culminado este proceso el personal encargado de la seguridad de la mina hace la lectura de la temperatura, cantidad de gas, y de otros factores que intervienen; además es el único que puede autorizar al personal para que ingrese al frente de trabajo.

\section{Dimensiones del Tajo}

- Longitud: 30m y Altura: 70m

- Potencia de la veta: $0.19 \mathrm{~m}$

- Inclinación: mayor a $60^{\circ}$

\section{Preparación}

Para la preparación de los tajos se trabajarán con las dimensiones que se muestran en los siguientes gráficos:

Gráfico 2: Galerías Principales (2m x 2,50m)

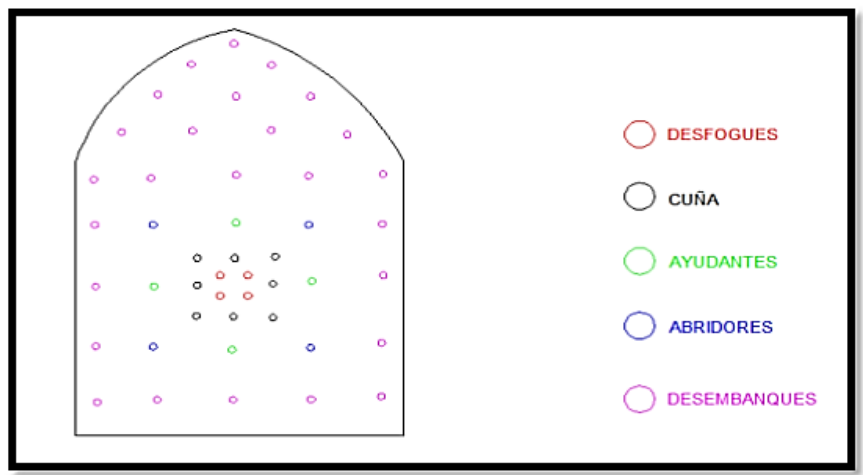

Fuente: Diego Chacha

Gráfico 3: Chimeneas de Acceso (1,80m x 1,20m)

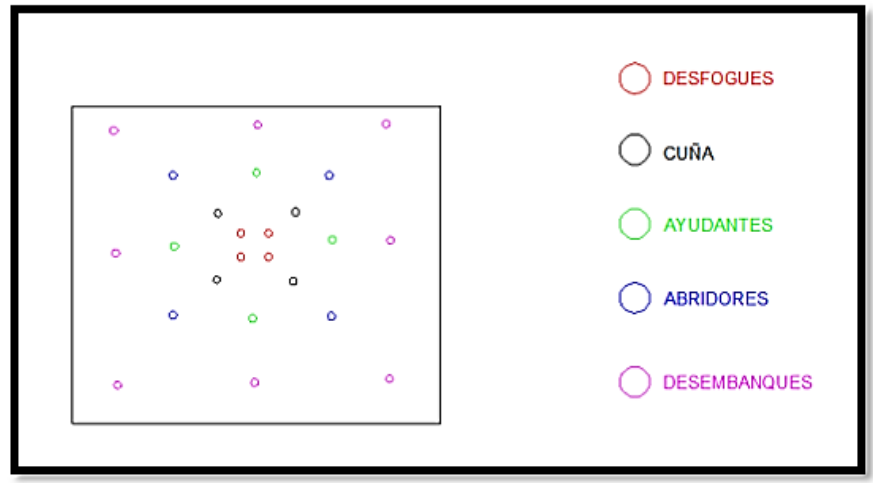

Fuente: Diego Chacha 
Gráfico 4: Ventanillas $(1,50$ x 1,20m)

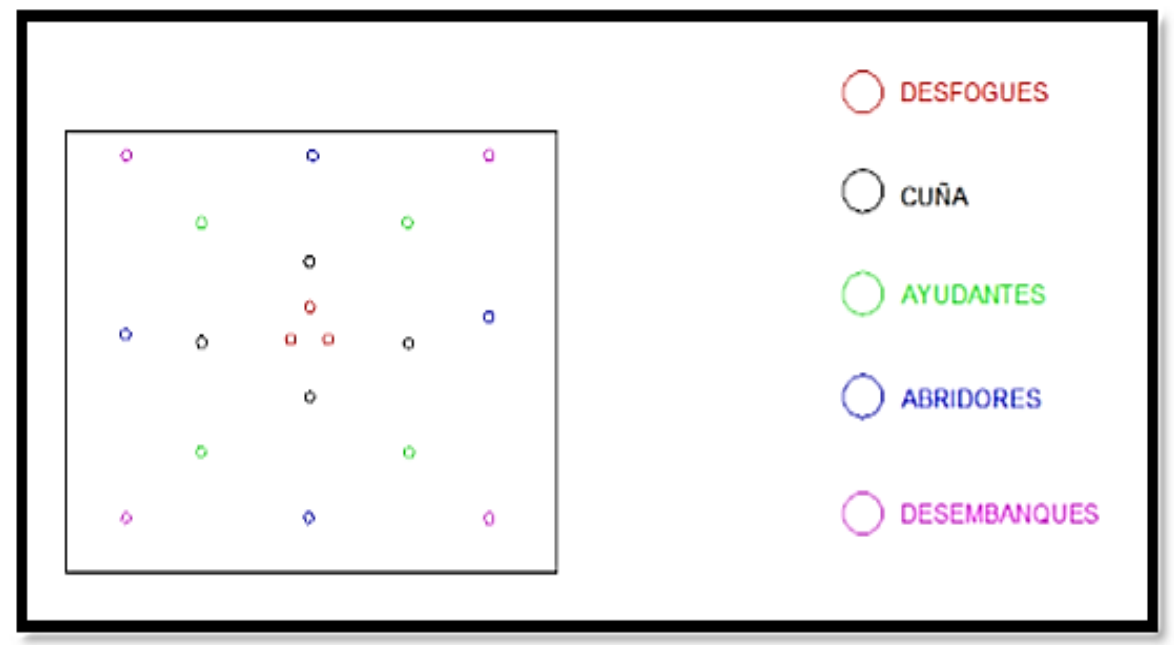

Fuente: Diego Chacha

Gráfico 5: Subniveles $(1,50 \mathrm{~m}$ x 3m)

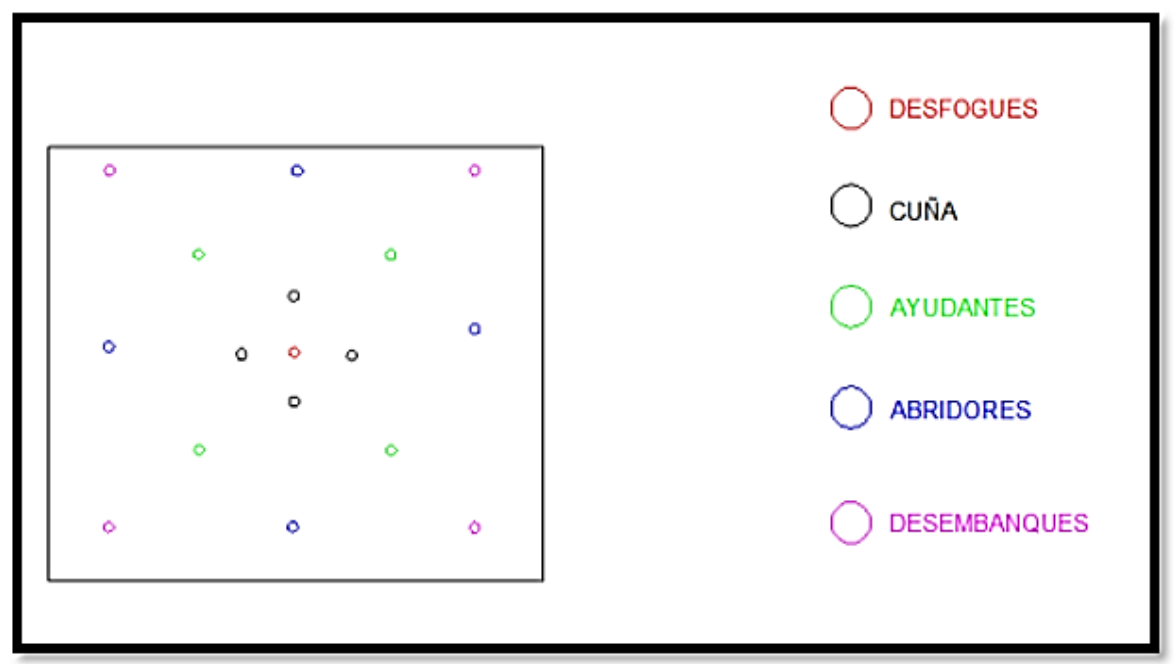

Fuente: Diego Chacha

\subsection{Diagnóstico del sistema de ventilación actual}

Cuenta con un sistema de ventilación forzada no planificada, con 2 turbinas portátiles que no se encuentran ubicadas con los debidos parámetros necesarios para un sistema de ventilación y mangas de ventilación que no están bien instaladas, además de una turbina principal ubicada en el crucero principal lo que da como resultado una ventilación deficiente. 
Figura 2: Galería sin Ventilación con Presencia de Gas

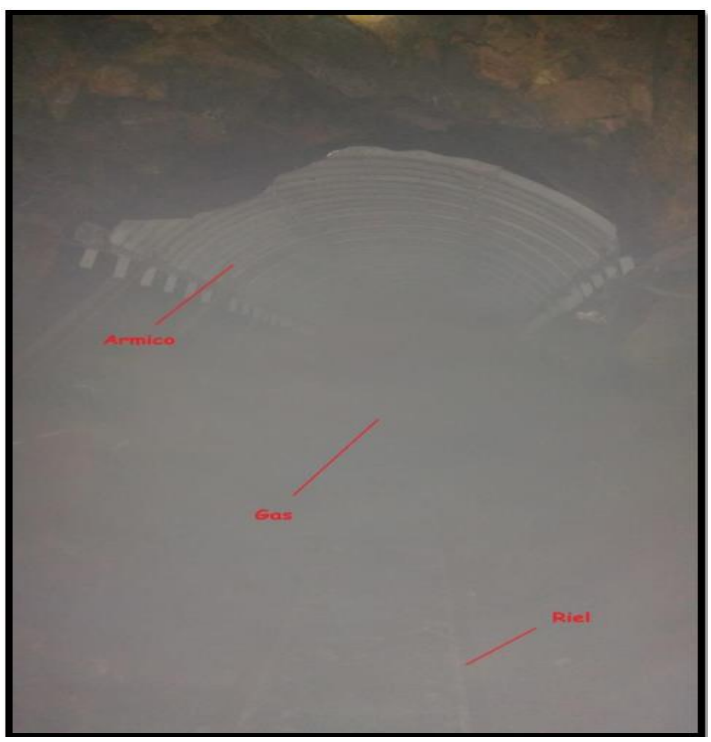

Fuente: Empresa Produmin S.A.

Este esquema de ventilación nos indica cual es la trayectoria que sigue el aire comprimido con el que se ventila las labores de preparación, operación, desarrollo y exploración en tajos; una vez culminada la etapa de perforación seguida de la etapa de carga de explosivos se deja abierta la tubería de aire para que al momento de la voladura los gases emitidos sean diluidos por el aire comprimido y sea más cómodo para los mineros el ingreso a los diferentes frentes de trabajo.

Gráfico 6: Esquema de Ventilación Actual

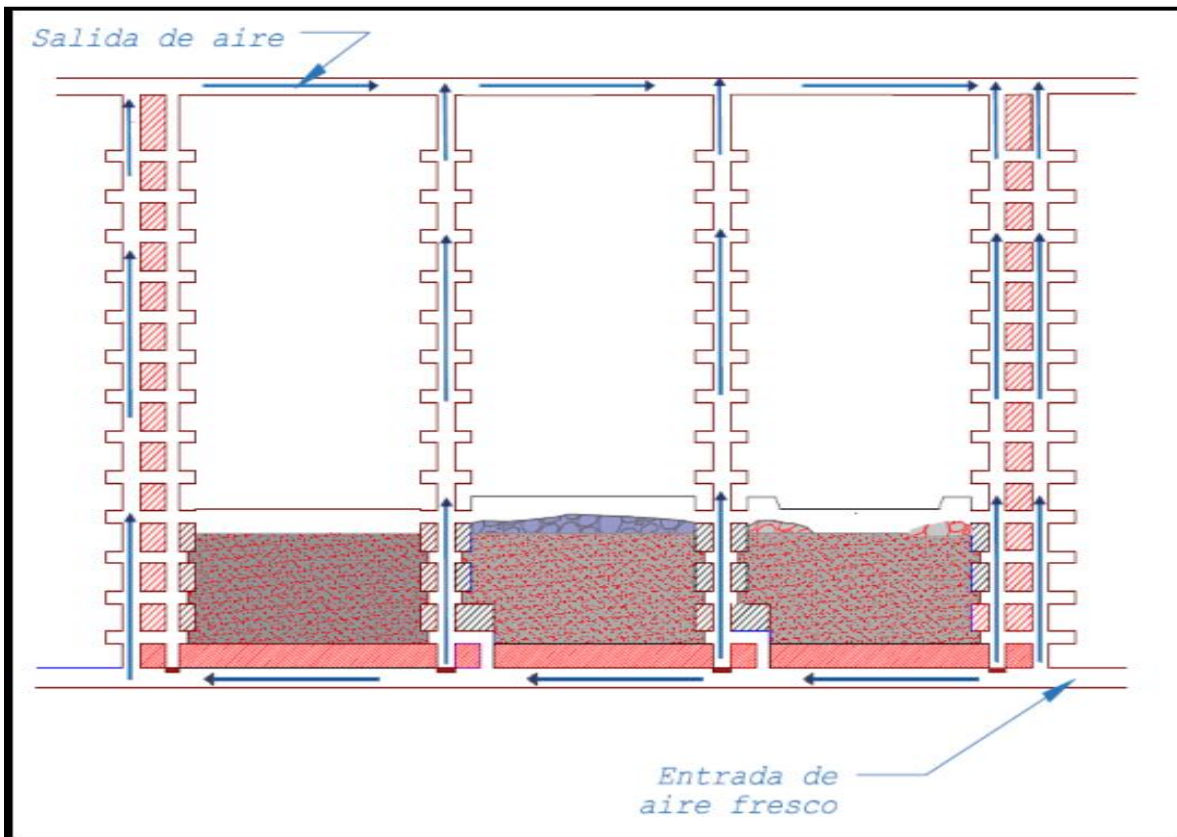

Fuente: Empresa Produmin S.A. 


\section{Requerimiento de aire por el personal}

Se trabaja con una cantidad de 25 personas entre perforistas, ayudantes y pateros. Para determinar el requerimiento de aire por el personal se necesita aplicar la siguiente fórmula:

$$
\mathrm{Q}_{1}=\mathrm{n} * \mathrm{q}
$$

En donde:

$\mathrm{Q}_{1}=$ cantidad de aire necesario para el " $\mathrm{n}$ " de personas $\left(\mathrm{m}^{3} / \mathrm{min}\right)$

$\mathrm{q}=$ Cantidad de aire mínimo por persona $\left(3 \mathrm{~m}^{3} / \mathrm{min}\right)$

$\mathrm{n}=$ Número de Personas en el Lugar

Por lo tanto, $\mathrm{Q}_{1}=75 \mathrm{~m}^{3} / \mathrm{min}$

\section{Requerimiento de aire por la cantidad de equipos en funcionamiento y polvo en suspensión}

Se trabaja con 10 perforadoras neumáticas, y los gases producidos debido a la fricción de $\mathrm{CO}_{2}$ es de $0,25 \mathrm{ppm}$. Para determinar el requerimiento de aire por la cantidad equipo que se tiene en funcionamiento y polvo en suspensión se aplica la siguiente fórmula:

$$
\mathrm{Q}_{2}=\mathrm{K} * \mathrm{~N}
$$

En donde:

$\mathrm{Q}_{2}=$ Cantidad necesaria para partículas desprendidas de equipos $\left(\mathrm{m}^{3} / \mathrm{min}\right)$

$\mathrm{K}=$ Cantidad de aire necesario por cada Operadora $\left(3 \mathrm{~m}^{3} / \mathrm{min}\right)$

$\mathrm{N}=$ Numero de equipos operando autorizados que trabajan en la mina.

Por lo tanto, $\mathrm{Q}_{2}=30 \mathrm{~m}^{3} / \mathrm{min}$

\section{Requerimiento por consumo de explosivos}

El cálculo se basa en los siguientes valores:

- Formación de $0,040 \mathrm{~m}^{3}$ de productos tóxicos por la voladura de $1 \mathrm{Kg}$. de explosivo.

- Tiempo de ventilación no mayor a 30min. Con excepción de explosivos en masa; es decir, sea el tiempo dentro del turno, que gasta el minero en regresar al frente después de haber realizado la voladura.

- De acuerdo a algunos reglamentos de seguridad, los productos tóxicos de la explosión deben ser diluidos a no más de $0,008 \%$ en volumen. 
Por consiguiente, para determinar el caudal de aire que se requiere llevar al frente de trabajo se debe aplicar la siguiente fórmula:

$$
\mathrm{Q}_{3}=100 * \mathrm{a} * \mathrm{~A} /(0,008 * \mathrm{t})
$$

En donde:

$\mathrm{Q}_{3}=$ cantidad de aire necesario en $\mathrm{m}^{3} / \mathrm{min}$

$\mathrm{A}=$ Cantidad de explosivo en la voladura en $\mathrm{Kg}$

$\mathrm{a}=0,040 \mathrm{~m}^{3} / \mathrm{Kg}$

$\mathrm{t}=$ tiempo de pausa entre la voladura y el regreso al frente o tiempo de ventilación en minutos

Por lo tanto, $\mathrm{Q}_{3}=16,67 \mathrm{~m}^{3} / \mathrm{min}$

\section{Requerimiento para mantener óptimas condiciones ambientales}

En interior mina trabajan 200 personas, el aire que ingresa tiene una velocidad de $9 \mathrm{~m} / \mathrm{min}$, los niveles más críticos son 50 y 30, y tienen un total de 7 niveles; asimismo trabajan a una temperatura de entre los 24 a $26^{\circ} \mathrm{C}$, estando ubicados en distintas áreas, además la línea principal tiene $2,40 \mathrm{~m}$ de alto por $2,10 \mathrm{~m}$ de ancho. Consecuentemente, para determinar la cantidad de aire que se requiere para mantener óptimas condiciones ambientales se aplica la siguiente fórmula:

$$
\mathrm{Q}_{4}=\mathrm{V} * \mathrm{n} * \mathrm{~A}
$$

En donde:

Q4= Cantidad de aire para mantener condiciones ambientales ideales

$\mathrm{V}=$ Velocidad del aire usando valores de $\mathrm{T}_{1}(9 \mathrm{~m} / \mathrm{min})$

$\mathrm{N}=$ Numero de niveles a trabajar con temperaturas elevadas

$\mathrm{A}=$ área promedio de la sección de labor

Por lo tanto, $\mathrm{Q}_{4}=317,52 \mathrm{~m}^{3} / \mathrm{min}$

\section{Total de CFM utilizando en interior mina}

Se extrae gases de las voladuras y se realiza el ingreso de aire puro con los compresores, los cuales envían aire puro en forma focalizada con un caudal de 3000cfm como mínimo, más el ciclo de turbinas automatizadas. Esto se da, cada que se produce una voladura para que por efecto de diferencia de presiones el aire recircule.

En cada frente de explotación se tiene la capacidad de enviar en tuberías de 1", las cuales proyectan un caudal máximo de $492,11 \mathrm{~m}^{3} / \mathrm{s}$. 
Tabla 1:Total de CFM y m³/min en Sistema Aspirante e Impelente

\begin{tabular}{|c|c|c|c|}
\hline \multicolumn{4}{|c|}{$\begin{array}{l}\text { TOTAL DE CFM Y m3/min SISTEMA ASPIRANTE E } \\
\text { IMPELENTE }\end{array}$} \\
\hline UBICACION & MOTOR & CFM & $\mathbf{m}^{3} / \mathbf{m i n}$ \\
\hline Crucero 557 & $5 \mathrm{HP}$ & 16000 & 453.26 \\
\hline Cecy Nivel Cero Norte & $5 \mathrm{HP}$ & 8000 & 226.63 \\
\hline Turbina Principal & 75HP & 38465 & 1089.67 \\
\hline Suma Total & & 62465 & 1769.54 \\
\hline
\end{tabular}

Fuente: Diego Chacha

\section{Resultados}

Tomando en cuenta los resultados de los cálculos realizados, se ha partido con el requerimiento de aire necesario de acuerdo al:

- Número de personas

- Polvo en suspensión

- Aumento de temperatura

- Consumo de explosivos

- Diseño de labor

Se determina que es conveniente implementar 4 turbinas secundarias de 5 hp ubicadas a 300m, una separada de la otra, teniendo en cuenta las siguientes reglas:

- La presión requerida es directamente proporcional a la longitud

- La presión es directamente proporcional al perímetro

Además, tomando en cuenta la distancia de la labor minera se determinó que es necesario utilizar un aproximado de $1200 \mathrm{~m}$ de manga de ventilación de 22”.

Figura 3: Turbina de 5Hp y Manga de Ventilación de 22"
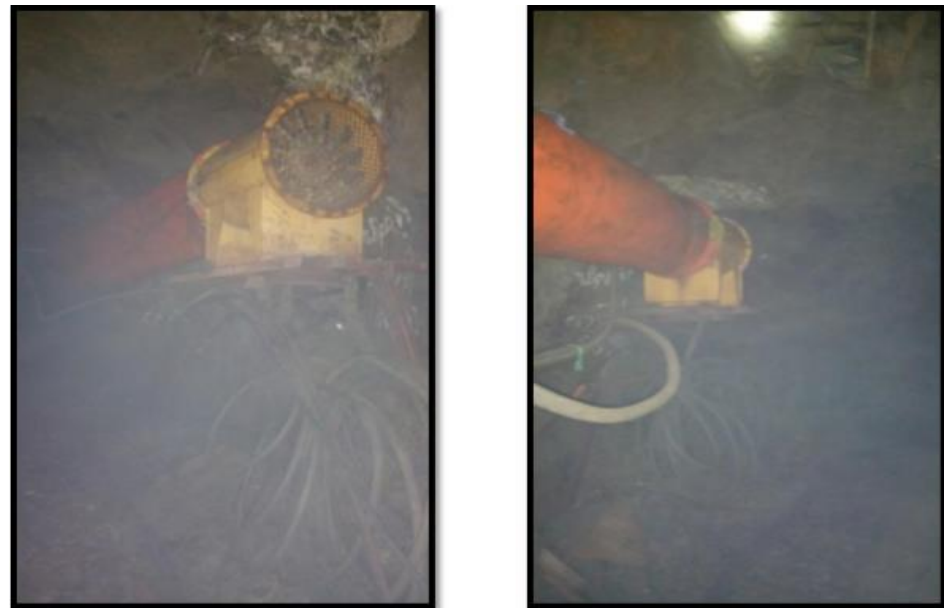

Fuente: Empresa Produmin S.A. 
En base, al resultado se realiza un análisis de factibilidad para la implementación del sistema de ventilación que requiere la empresa PRODUMIN S.A. para efectuar de forma segura los trabajos interior mina, de la misma manera se realiza el costo que tiene este sistema.

Tabla 2: Análisis de Factibilidad para la Implementación del Sistema de Ventilación

\section{Análisis de factibilidad para la Implementación del Sistema de Ventilación}

\begin{tabular}{|c|c|c|c|c|c|c|}
\hline $\begin{array}{l}\text { Número de disparos } \\
\text { actual }\end{array}$ & $\begin{array}{l}\text { Disparos/ } \\
\text { día }\end{array}$ & 1 & & & & \\
\hline $\begin{array}{l}\mathrm{N}^{\circ} \text { de disparos con } \\
\text { ventilación mecánica }\end{array}$ & $\begin{array}{l}\text { Disparos/ } \\
\text { día }\end{array}$ & 3 & & & & \\
\hline Avance total & $\mathrm{m}$ & 288 & & & & \\
\hline Costo de avance & $\begin{array}{l}\text { Usd/m } \\
\text { (lineal) }\end{array}$ & 356,95 & & & & \\
\hline $\begin{array}{l}\text { Costo de transporte a } \\
\text { superficie }\end{array}$ & Usd & 6588 & & & & \\
\hline Costo de avance & Usd & 102801,60 & & & & \\
\hline $\begin{array}{l}\text { Valor en inversión del } \\
\text { circuito de ventilación }\end{array}$ & Usd & 42586,86 & & & & \\
\hline $\begin{array}{l}\text { Ganancia de Mineral por } \\
\text { exp-des }\end{array}$ & Ton & 835,80 & & & & \\
\hline Gr Finos & $\mathrm{Gr}$ & 20895 & & & & \\
\hline Valor mineral ganado & $\mathrm{Usd} / \mathrm{Gr}$ & 34,14 & & & & \\
\hline Ley mineral & $\mathrm{Gr} / \mathrm{ton}$ & 25 & & & & \\
\hline Potencia de la veta & $\mathrm{cm}$ & $<30$ & & & & \\
\hline Buzamiento & & $70^{\circ}$ & & & & \\
\hline \multirow[t]{2}{*}{ Costo de producción } & Usd/ton & 469,08 & & & & \\
\hline & & & \multicolumn{3}{|c|}{ Flujo Neto de fondos } & \\
\hline Mes & & & Abril & Mayo & Junio & Julio \\
\hline Inversión & & 151976,46 & & & & \\
\hline Ton cubicadas & & & 189 & 201,6 & 214,2 & 231 \\
\hline Gr finos & & & 4725 & 5040 & 5355 & 5775 \\
\hline $\begin{array}{l}\text { Ventas en el mineral } \\
\text { cubicado }\end{array}$ & & & 1615000,50 & 172267,20 & 183033,90 & 197389,50 \\
\hline Costo de producción & & & 88656,12 & 94566,53 & 100476,94 & 108357,48 \\
\hline Flujo neto de fondos & & & 728444,38 & 77700,67 & 82556,96 & 89032,02 \\
\hline tasa de interés & & $15 \%$ & & & & \\
\hline VAN & & 315830,1 & & & & \\
\hline TIR & & $37 \%$ & & & & \\
\hline
\end{tabular}

Fuente: Diego Chacha. 
Tabla 3: Datos Técnicos

\begin{tabular}{|c|c|c|c|c|}
\hline \multicolumn{5}{|l|}{ Datos Técnicos } \\
\hline \multicolumn{5}{|l|}{ Modelo } \\
\hline \multicolumn{3}{|c|}{ Motor del ventilador } & 7,00 & HP \\
\hline \multicolumn{3}{|c|}{$\mathrm{N}^{\circ}$ ventiladores } & 4,00 & Unid. \\
\hline \multicolumn{3}{|c|}{ Caudal del ventilador } & 8000,00 & CFM \\
\hline \multicolumn{3}{|c|}{$\%$ Eficiencia } & 7040,00 & CFM \\
\hline \multicolumn{3}{|l|}{ Caudal total } & 28160,00 & CFM \\
\hline \multicolumn{3}{|c|}{ Caudal requerido en el frente de trabajo } & 14400,00 & CFM \\
\hline \multicolumn{3}{|c|}{ Funcionamiento equipo } & 8,00 & horas \\
\hline \multicolumn{3}{|c|}{ Total Horas funcionamiento } & 54067200,00 & pies $^{3}$ dia \\
\hline PARÁMETROS & UND & $\begin{array}{c}\text { COSTO } \\
\text { UNITARIO }\end{array}$ & CANTIDAD & $\begin{array}{l}\text { TOTAL } \\
\text { (USD) }\end{array}$ \\
\hline Ventilador & US\$/UND & 5000,00 & 4 & 20000,00 \\
\hline $\begin{array}{l}\text { Tableros } \\
\text { Eléctricos }\end{array}$ & US\$/UND & 491,34 & 4 & 1965,37 \\
\hline $\begin{array}{l}\text { Manga de } 22 " \mathrm{e} \\
\text { Instalación }\end{array}$ & $\mathrm{US} \$ / \mathrm{m}$ & 9,44 & 1200 & 11328,00 \\
\hline $\begin{array}{l}\text { Armado e } \\
\text { Instalado del } \\
\text { Arranque }\end{array}$ & US\$ & 199,66 & 4 & 798,66 \\
\hline $\begin{array}{l}\text { Cable } \\
\text { Concéntrico } \\
3 \times 10\end{array}$ & USD & 3,00 & 1200 & 3595,46 \\
\hline SUBTOTAL & USD & & & 37687,49 \\
\hline Gastos generales & $10 \%$ & & & 3768,75 \\
\hline Imprevistos & $3 \%$ & & & 1130,62 \\
\hline TOTAL & USD & $5.500,78$ & 1212 & 42586,86 \\
\hline TOTAL & USD/CFM & 1,51 & & \\
\hline TOTAL & USD/pies ${ }^{3}$ _dia & 0,0007 & & \\
\hline
\end{tabular}

Fuente: Elaborado por Diego Chacha

\section{Conclusiones}

- La compañía PRODUMIN S.A. tiene un diseño tradicional de ventilación forzada de mina; mediante dos turbinas auxiliadas con ventilación natural; este sistema demuestra deficiencia conforme ha ido avanzando el frente de extracción minera; durante una voladura se genera los siguientes gases con sus respectivas concentraciones promedios: $\mathrm{NO} 2$ a $0,98 \mathrm{ppm}$; $\mathrm{CO} 2$ a $4,9 \%$; $\mathrm{CO}$ a $0.9 \mathrm{ppm}$; de un volumen de $0,040 \mathrm{~m}^{3}$ por $1 \mathrm{Kg}$. de explosivo, considerando un tiempo de evacuación 
de 30 minutos a comparación del tiempo de deyección tradicional de 12 horas, se necesitaría un caudal de $16,67 \mathrm{~m} 3 / \mathrm{min}$ en generación de aire auxiliada con ventilación aspirante para evitar minúsculos residuos en la sección como pequeñas acumulaciones en labores secundarias.

- El caudal de aire forzado que ingresa al tajo a fin de ventilar la construcción minera (unitaria) durante el trabajo con el fin de garantizar el buen desempeño del personal conformado por 3 obreros se determina un caudal de $60 \mathrm{~m}^{3} / \mathrm{min}$, en cuanto al funcionamiento de los equipos de perforación conformado por 3 perforadoras se estipula que debe contener un caudal de $30 \mathrm{~m} 3 / \mathrm{min}$. En tanto a las condiciones normales de la mina con 200 hombres trabajando se necesitaría un caudal aire de $317,52 \mathrm{~m} 3 / \mathrm{min}$. con una velocidad de ingreso de $9 \mathrm{~m} / \mathrm{min}$ a los niveles.

- Se determinó que el sistema de ventilación debe estar conformado por 4 turbinas secundarias de $5 \mathrm{hp}$ ubicadas a $300 \mathrm{mts}$. de distancia entre cada una de ellas; considerando la distancia de la labor minera se estableció que se utilizara un aproximado de $1200 \mathrm{mts}$. de manga de ventilación de 22”.

- El Costo de la implementación del Sistema de Ventilación es de 42.586,86 reflejado en el mejoramiento de la productividad, ya que no habrá paras innecesarias las cuales acortan los tiempos de despeje de gases nocivos.

\section{Referencias Bibliográficas}

Álzate, R. (2005). Guía metodológica para ventilación subterránea de minas. Universidad Tecnológica del Choco Diego Luis Córdova. Departamento de Ingeniería de Minas y Ambiental. Facultad de Ingeniería. Colombia

Andrade, S. (2002). Guía metodológica de seguridad para ventilación de minas.

Recuperado de: https://www.sernageomin.cl/wpcontent/uploads/2018/12/200812GuiaVentilacionMinas.pdf

ARCOM. (2014). Reglamento de seguridad minera. Registro Oficial No. 247, de 16 mayo de 2014. Quito, Ecuador.

Babilon, D. (2008). Ventilación minera. Recuperado de: https://www.horizonteminero.com/ventilacion-minera/

Baldock, J (1982). Geología del Ecuador. División de Investigación Geológico Minera. Quito, Ecuador.

Campillos, A. (2015). Optimización y modelización del circuito de ventilación de una mina subterránea.

Recuperado

de: http://oa.upm.es/36496/1/PFC_Alberto_Campillos_Prieto.pdf

Carrasco Galán, J. (2002). Manual de ventilación de minas y obras subterráneas. Recuperado de: http://geco.mineroartesanal.com/tiki-download_wiki_attachment.php?attId=637 
Córdova, C. \& Molina, J. (2011). Caracterización de sistemas de ventilación en minería subterránea. http://www.revistas.unal.edu.co/index.php/rbct/article/view/29252/39426

Jiménez, P. (2011). Ventilación de minas subterráneas y túneles. Recuperado de: http://www.iimp.org.pe/website2/jueves/ultimo261/jm20111110_libro.pdf

Rojas, S. (2010). Ventilación de minas subterráneas. Universidad Nacional Daniel Alcides Carrión. Escuela de Formación Profesional de Minas. Facultad de Ingeniería. Cerro de Pasco, Perú.

Vega, D. (2007). El origen de polvos y su mitigación en la minería. Recuperado de: https://www.monografias.com/trabajos53/contaminacion-polvosmineria/contaminacion-polvos-mineria2.shtml

McPherson, M.J. (2009) Ingeniería de Ventilación Subterránea, Mine Ventilation Services, Inc.

Díaz, M. (2009) Salud y Seguridad en trabajos de minería. Buenos Aires, Argentina. Aulas y Andamios Editora.

Carrasco, J. (2012) Manual de Ventilación de Minas y Obras Subterráneas. Madrid, España; Aitemin

Luque, V. (1990) Manual de Ventilación de Minas; Madrid, España. Asociación de Investigación Tecnológica de Equipos Mineros España 
PARA CITAR EL ARTÍCULO INDEXADO.

Mejía Flores, M. A., Morales Chacha, K. M., \& Chacha Borja, D. R. (2020). Sistema de ventilación para labores subterráneas de la empresa PRODUMIN S.A. ConcienciaDigital, 3(2.2), 154-168. https://doi.org/10.33262/concienciadigital.v3i2.2.1253

\section{【 Clencia}

El artículo que se publica es de exclusiva responsabilidad de los autores y no necesariamente reflejan el pensamiento de la Revista Conciencia Digital.

El artículo queda en propiedad de la revista y, por tanto, su publicación parcial y/o total en otro medio tiene que ser autorizado por el director de la Revista Conciencia Digital.
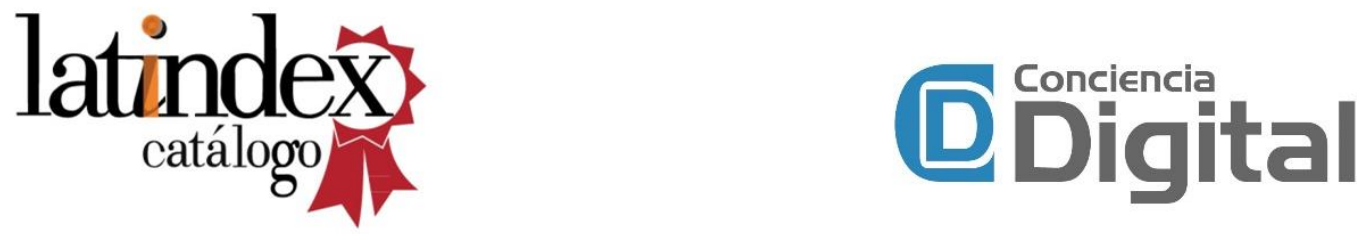